\title{
The Integrated Analysis of Differential Long Non- Coding RNAs Expression Profiles in Extracellular Vesicles Derived From Chemoradioresistant and Parental Nasopharyngeal Carcinoma Cells
}

\section{Wei Xiong}

The Third Affiliated Hospital of Kunming Medical University: Yunnan Cancer Hospital

\section{Runmei Sun}

The Third Affiliated Hospital of Kunming Medical University: Yunnan Cancer Hospital Chunyan Li

The Third Affiliated Hospital of Kunming Medical University: Yunnan Cancer Hospital Cheng Li

The Third Affiliated Hospital of Kunming Medical University: Yunnan Cancer Hospital Jiyong Qin

The Third Affiliated Hospital of Kunming Medical University: Yunnan Cancer Hospital Qiuyan Liu

The Third Affiliated Hospital of Kunming Medical University: Yunnan Cancer Hospital

\section{Xiaoli Wang}

The Third Affiliated Hospital of Kunming Medical University: Yunnan Cancer Hospital

\section{Qiaoli Wang}

The Third Affiliated Hospital of Kunming Medical University: Yunnan Cancer Hospital

\section{Guoqiang Xu}

The Third Affiliated Hospital of Kunming Medical University: Yunnan Cancer Hospital

\section{Ruixue Cao}

The Third Affiliated Hospital of Kunming Medical University: Yunnan Cancer Hospital

\section{Chuanzheng Sun}

The Third Affiliated Hospital of Kunming Medical University: Yunnan Cancer Hospital Yi Yang ( $\sim$ ynszlyyyangyi@sohu.com )

The Third Affiliated Hospital of Kunming Medical University: Yunnan Cancer Hospital https://orcid.org/0000-0002-6114-9955

\section{Research Article}


Keywords: Nasopharyngeal carcinoma, Extracellular vesicles, Long non-coding RNAs, Chemoradiotherapy, Migration, Invasion

Posted Date: August 10th, 2021

DOl: https://doi.org/10.21203/rs.3.rs-779650/v1

License: (c) (1) This work is licensed under a Creative Commons Attribution 4.0 International License. Read Full License 


\section{Abstract}

The patients with nasopharyngeal carcinoma (NPC) suffer from poor outcomes after chemoradiotherapy. Extracellular vesicles (EVs) play crucial roles in regulating cancer progression and chemoradioresistance. To investigate the functional role of chemoradioresistant cell-derived EVs for parental cells, and long noncoding RNAs (IncRNAs) expression profiles in EVs secreted by chemoradioresistant cells. The effect of chemoradioresistant NPC cell-derived EVs on migration and invasion abilities of parental cells were detected by Transwell assays. Human cancer IncRNA PCR array was performed on EVs released from chemoradioresistant NPC cells (CNE1R and CNE2R) and parental cells (CNE1 and CNE2). The concurrent chemoradioresistance of CNE1R and CNE2R was significant higher than that of CNE1 and CNE2. Transmission Electron Microscopy (TEM), Nanoparticle Tracking Analysis (NTA), and western blotting results showed the CNE1, CNE2, CNE1R and CNE2R -derived EVs were successfully obtained. The chemoradioresistant cell-derived EVs remarkably promoted migration and invasion ability of parental cells with different differentiation. Notably, a total of 91 differentially expressed (DE) IncRNAs were regulated in EVs by chemoradiotherapy. Among them, the 4 upregulated (MALAT1, FAM212B-AS1, LINCPINT and H19) and 7 down-regulated (SNHG16, CDKN2B-AS1, ZFAS1, CCAT1, SNHG6, GAPLINC and TUG1) DE IncRNAs associated with chemoradioresistance, were identified in EVs derived from chemoradioresistant cells with different differentiation. We found the EVs derived from chemoradioresistant NPC cells regulated an aggressive phenotype of NPC cells, and identified an altered IncRNAs expression pattern in chemoradioresistant NPC cell- derived EVs.

\section{Introduction}

Nasopharyngeal carcinoma (NPC) belongs to a squamous-cell carcinoma that located in the nasopharyngeal epithelium [1]. This carcinoma has a distinctive ethnic and geographic distribution, which has higher occurrence in southern China than in the rest of the world [2]. EBV infection and lymphoepithelial-like histologic are notable characteristics of NPC [2]. Non-keratinizing, undifferentiated NPC (WHO type III) is the is the most common subtype, and accounts for $63-95 \%$ of all NPC cases worldwide [3]. Prognosis is poor once the NPC cells migrate even if this carcinoma obtained advanced therapies [4]. The metastatic NPC is mainly caused by many factors such as Epstein-Barr virus infection, heredity and environmental factors $[5,6]$. These factors can result in disruption of cell junctions, epithelial-mesenchymal transition (EMT), invasion, and clonogenicity [7]. Although the application of radiotherapy or chemotherapy techniques have contributed to the improvement in the local control of NPC, chemoradioresistance is a major impediment to achieve longterm survival [8]. A majority of the NPC patients receiving radiotherapy treatment confront with local recurrence and distant metastasis within 1.5 years for radioresistance [9].

Long non-coding RNAs (IncRNAs) are a class of non-protein-coding transcripts above 200 nucleotides in length [10]. LncRNAs can function as competing endogenous RNAs and antisense RNAs, and are proved to act as either promoters or suppressors in various carcinomas. The IncRNAs mainly act in epigenetic level, transcriptional level and posttranscriptional level, among other processes [11]. The recent studies 
have showed the IncRNAs are abnormal various types of cancers treated with chemoradiotherapy. For instance, IncRNAs with aberrant expression values between the two groups of LARC patients have been identified in local advanced rectal cancer (LARC) with different neoadjuvant chemoradiotherapy downstaging depth score [12]. Wei Xiong et al has identified 2,662 differentially expressed IncRNAs in 5FU-based colorectal cancer HCT116 cells relative to those in parental HCT116 [13] However, the abnormal IncRNA expression profile in NPC receiving chemoradiotherapy, and in released EVs of that is still known few.

Extracellular vesicles (EVs) released from various types of cells, can be secreted to the extracellular space through fusion with plasma membrane, and contribute to intercellular communication [14]. Classically, EVs can be further classified on the basis of their biogenesis, size and biophysical properties, i.e., exosomes, microvesicles, and apoptotic bodies [15]. EVs carry amounts of bioactive proteins, mRNA, small RNA and lipids, and transfer their contents to recipient cells to involve in tumor cell progress [16], can play crucial role in phenotype alteration of sensitive cancer cells. For instance, NPC exosomes can regulate biological behaviour of NPC cells $[17,18]$. MiR-9 exists in human 5-8F cell-derived exosomes [19]. Decrease of miR-9 expression via promoter methylation results in increase of CXCR4 expression, stimulating biological behavior (e.g. proliferation, migration and invasion) of NPC cells via the activation of MAPK pathway [20]. NPC exosomes modulates epithelial-mesenchymal transition of cells via delivering mitotic cytokines (e.g., TGF- $\beta$ ), resulting in resistance to chemotherapy [21]. However, the IncRNA expression profile from NPC-derived EVs is wanting. Whether the chemoradiotherapy-induced NPC EVs regulate aggressive phenotypes of NPC cells is on the way.

In our study, we explored the functional role of chemoradioresistant NPC cells-derived EVs on parental NPC cells with different differentiation, and for the first time identified differentially expressed (DE) IncRNAs related with chemoradioresistance in chemoradioresistant NPC cell-derived EVs via PCR array. The effect of chemoradioresistant NPC cell-derived EVs on migration and invasion abilities of parental cells with different differentiation were evaluated. The shared IncRNAs associated with chemoradioresistance were further identified in EVs derived from chemoradioresistant cells with different differentiation.

\section{Material And Methods}

\section{Cell culture}

Human NPC cell lines CNE-1 (well differentiation) and CNE-2 (poor differentiation) used in this study were obtained from China Center for Type Culture Collection (Wuhan, China), cultured in RPMI-1640 medium (GIBCO), added with $10 \%$ fetal bovine serum (FBS), $100 \mathrm{U} / \mathrm{ml}$ penicillin and $100 \mathrm{mg} / \mathrm{ml}$ streptomycin (Invitrogen, Carlsbad, CA, USA) in $5 \% \mathrm{CO} 2$ at $37^{\circ} \mathrm{C}$.

\section{Establishment of concurrent chemoradioresistant NPC cell lines}


When CNE-1 and CNE-2 cells were up to the confluence of $60 \%$, they were cultured in the complete medium containing $0.05 \mathrm{ug} / \mathrm{mL}$ cisplatin, and were exposed to $6 \mathrm{~Gy}$ irradiation using a 6-MV X-ray linear accelerator (ELEKTA, Beijing, China) at $200 \mathrm{cGy} / \mathrm{min}$ dose rate. After $24 \mathrm{~h}$, the cells were cultured in the fresh complete medium containing $0.05 \mathrm{ug} / \mathrm{mL}$ cisplatin (Sigma-Aldrich, Saint Louis, MO, USA). When the cell density reached $80-90 \%$, they were further passaged and cultured. The next chemoradiotherapy was performed when the cell growth was in stable state for a total of 10 times. The CNE-1 and CNE-2 cell lines treated with chemoradiotherapy were named as CNE1R and CNE2R, respectively.

\section{Colony formation assay}

Each cell (CNE-1, CNE-2, CNE1R and CNE2R) were respectively inoculated in 6-well plates at a density of $300,400,800,1000,2000,4000$ cells/well and cultured in $200 \mu \mathrm{L}$ cell culture medium for $24 \mathrm{~h}$.

Subsequently, the cells were removed the original medium and cultured in the fresh complete medium containing $0.05 \mathrm{ug} / \mathrm{mL}$ cisplatin. At the same time, these cells were respectively treated with gradient doses of irradiation ( $0 \mathrm{~Gy}, 2 \mathrm{~Gy}, 4 \mathrm{~Gy}, 6 \mathrm{~Gy}, 8 \mathrm{~Gy}$ and $10 \mathrm{~Gy}$ ) according to the gradient cell numbers under the above radiation condition. After 14 days, the cells were washed, fixed for $30 \mathrm{~min}$ using methanol, and stained with $0.1 \%$ crystal violet (Sigma, MO, USA) for $10 \mathrm{~min}$. The number of clones $>50$ was counted under a microscope (DMIRB, Leica, Germany) to calculate the plating efficiency (PE) (\%) that is equal to (number of colonies/inoculated cell number) $\times 100 \%$. Surviving fraction (SF) that is equal to number of colonies/ (number of cells seeded $\times \mathrm{PE}$ ) $\times 100 \%$. We plotted the cell survival curve using the multi-target

single-hit model: $\mathrm{SF}=1-\left(1-\mathrm{e}^{-\mathrm{D} / \mathrm{D} 0}\right)^{\mathrm{N}}$, and the radiobiological parameters $\mathrm{D} 0$ (the dose that gave an average of one hit per target), Dq (quasi-threshold dose) and SF2 (the SF following exposure to 2 Gy radiation) were determined using the survival curve. The experiments were repeated in triplicate.

\section{EV isolation, quantitation and characterization}

EVs isolation and identification referred to the MISEV2018 guidelines [22]. NPC cells were cultured until $80 \%-90 \%$ confluence was reached, and then changed with serum starvation. After incubation for $48 \mathrm{~h}$, the supernatants were centrifuged at $300 \mathrm{~g}$ for $10 \mathrm{~min}$ to remove dead cells and cell debris. Subsequently, the supernatants were filtered using a $0.22 \mu \mathrm{m}$ membrane and concentrated to $1 \mathrm{ml}$ using Amicon Ultra 15 Centrifugal Filter Unit (Millipore, USA). The lipids left was transferred to a fresh microcentrifuge tube. EVs were isolated using the ExoQuick TC kit (System Biosciences, USA) referring to manufacturer's protocol. The isolated EVs were resuspended in PBS. The EVs were fixed using $4 \%$ paraformaldehyde and loaded onto a TEM copper grid (Agar Scientific Ltd., Stansted, UK), and then washed with $1 \%$ glutaraldehyde, PBS, and distilled water., Next, EVs were incubated with $4 \%$ uranyl acetate and observed under TEM (Hitachi H7500 TEM, Tokyo, Japan).

Nanoparticle tracking analysis (NTA) was performed by Nanosight (ZetaView PMX 110, Particle Metrix, Meerbusch, Germany, ZetaView 8.04.02 SP2) to characterize the size distribution and concentration of EVs derived from CNE1, CNE2, CNE1R and CNE2R.

\section{Western Blotting}


EV protein quantification was obtained using Pierce BCA Protein Assay Kit (Life Technologies, USA) following the manufacturer's instructions.. The proteins were separated by $10 \%$ SDS-PAGE and transferred to nitrocellulose membranes. Membranes were incubated with primary antibodies for mouse anti-CD63 (Cat\# ab108950, Abcam, 1:1000) and GAPDH (Proteintech, Chicago, UK; 1:1000) at $4^{\circ} \mathrm{C}$ overnight, and then washed and incubated with secondary antibody (Santa Cruz, Dallas, USA) for 2 hours. Protein bands were visualized using enhanced chemiluminescence reagent (ECL AdvanceTM; GE Healthcare) and images were obtained via a ChemiDoc MP system (Bio-Rad, Hercules, CA, USA).

\section{EV Labelling and Internalization}

EVs were isolated from chemoradioresistant NPC cells $\left(1.5 \times 10^{6}\right.$ cells), and suspended in $100 \mathrm{ul} 1 \times \mathrm{PBS}$, and then were mixed with $1 \mathrm{ul}$ Dil lipophilic dye (Invitrogen, Carlsbad, CA, USA) for 4 min at $25^{\circ} \mathrm{C}$. After adding $1 \mathrm{ml}$ FBS to terminate the reaction, the 200ul ExoQuick-TC was added into mixture on ice for incubating $30 \mathrm{~min}$. The labelled EVs were reisolated by ultracentrifugation at $14000 \mathrm{rpm}$ at $4^{\circ} \mathrm{C}$ for $3 \mathrm{~min}$. Recipient CNE1 and CNE2 cells were incubated with $300 \mathrm{ul}$ Dil-labelled EVs for $3 \mathrm{~h}$ at $37^{\circ} \mathrm{C}$ in $5 \% \mathrm{CO} 2$, fixed using $4 \%$ paraformaldehyde at $25^{\circ} \mathrm{C}$ for $10 \mathrm{~min}$, washed, and incubated with DAPI (Invitrogen, Carlsbad, CA, USA) for 10 minutes at $25^{\circ} \mathrm{C}$, and subjected to fluorescence microscope (Nikon, TE300) with $40 \times$ magnification $(n=3)$.

\section{Migration and invasion assays}

For the migration assays, $1 \times 10^{5}$ cells were seeded onto the upper chamber (Cat\#353097, FALCON,) added with $500 \mu \mathrm{l}$ of serum-free DMEM and incubated with or without EVs. Subsequently, the $700 \mu \mathrm{l}$ DMEM with $10 \%$ FBS was supplemented into the lower chambers. After $24 \mathrm{~h}$ of incubation, the cells underneath the membrane were fixed using $4 \%$ paraformaldehyde and stained with $800 \mu$ crystal violet (Cat\# C0121; Beyotime, Shanghai, China) for $30 \mathrm{~min}$ at room temperature. The number of migrated cells was counted under a microscope. For the invasion assays, the basic steps were the same as above, except that chambers precoated with matrigel (Cat \#354480, BioCoat,).

\section{The RNA extraction, cDNA Synthesis Using an RT2 First Strand Kit and human cancer IncRNA PCR array}

Total RNA derived from NPC cells was obtained using Trizol reagent (Invitrogen, Carlsbad, CA, USA), and its concentration was measured by Nanodrop 2000 (Tiangen, Beijing, China). RNA were performed with agarose electrophoresis for RNA quality control. The cDNA was synthesized using $\mathrm{RT}^{2}$ first strand kit by reference to the manufacturer's manual (Cat:330401, Qiagen, Hilden, Germany). The $10 \mu$ reverse transcription mixture was prepared and thoroughly mixed with the genomic elimination mixture $(10 \mu \mathrm{l})$ and performed an incubated at $42^{\circ} \mathrm{C}$ for $15 \mathrm{~min}$. After the reaction termination at $95^{\circ} \mathrm{C}$ for $3 \mathrm{~min}$, a $91 \mu \mathrm{l}$ of RNase-free water was added into each reaction mixture and mixed for following assays.

The RT ${ }^{2}$ SYBR Green Master mix was used for human cancer IncRNA PCR array (Yingbiotech, Shanghai, China). Briefly, a real-time PCR mixture was prepared by adding the $650 \mu \mathrm{l}$ of 2 X PCR master mix, $102 \mu \mathrm{l}$ diluted cDNA, and $548 \mu \mathrm{l}$ RNase free water. The membrane on the PCR Array was carefully, and $10 \mu \mathrm{l}$ of 
the PCR mixture was added to each of the wells. Finally, PCR Array was performed in triplicates. GAPDH was used as endogenous control. The $2^{-\Delta \Delta C t}$ method was applied to calculate the relative IncRNAs expression.

\section{Statistical analysis}

All data were expressed as mean \pm SD in three duplicate experiments. GraphPad Prism 8 (GraphPad Software, Inc.) was applied for statistical analysis. Student's T-test was utilized to calculate the differences. The statistically significant differences were considered at $p<0.05$.

\section{Results}

\section{Identification of the chemoradioresistant CNE1R and CNE2R cells}

To identify whether CNE1R and CNE2R cells were resistant to concurrent chemoradiotherapy compared to parental cells, we performed colony formation assay. The results showed that the morphology of CNE1R and CNE2R cells presented the cell body elongated, the extended pseudopod and large intercellular space compared with the CNE1 and CNE2 cells (Fig. 1A). With the increase of radiation dose, the number of cell colonies significantly decreased. At the same radiation dose, the number of CNE1 and CNE2 cell colonies were lower than that of CNE1R and CNE2R cell colonies, respectively (Fig. 1B). The SF value of CNE1R and CNE2R was higher than that of CNE1 and CNE2, respectively (Fig. 1C). Additionally, there was an increase in D0, Dq and SF2 in the CNE1R and CNE2R relative to the CNE1 and CNE2, respectively (Table I), indicating an obvious chemoradioresistance were found in CNE1R and CNE2R as compared to CNE1 and CNE2. These results showed that the chemoradioresistanct NPC cells with different differentiation were successfully obtained.

\section{Identification of the chemoradioresistant and parental NPC cell -derived EVs}

To identify the collected EVs derived from the well and poorly differentiated NPC cells receiving with and without chemoradiotherapy, TEM and NTA were performed. As presented in Fig 2A and 2B, the obtained particles were consistent with EVs in size (100-150 nm) and morphology (round-shaped).

The concentration of CNE1-EVs, CNE1R-EVs, CNE2-EVs and CNE2R-EVs cells were 7.8E+9 Particles / $\mathrm{mL}, 1.3 \mathrm{E}+12$ Particles / mL, 1.2E+12 Particles / mL, 1.3E+12 Particles / mL, respectively. There were no remarkable differences between the concentration of released EVs of these cells except for CNE1EVs. Additionally, chemoradioresistant and parental NPC cell-derived EVs expressed its marker (CD63) protein. The above results revealed we successfully obtained EVs derived from chemoradioresistanct NPC cells with different differentiation (Fig. 2C).

\section{The chemoradioresistant NPC cells released- EVs potentiated migration and invasion ability of parental cells}


To clarify the functional roles of chemoradioresistant cell-released EVs in parental cells, we first identify wthether chemoradioresistant NPC cell -secreted EVs were efficiently taken up by parental cells. As expected, CNE1 and CNE2 taken up Dil-labelled EVs derived from CNE1R and CNE2R (Fig. 3). The further results showed that CNE1R-EVs and CNE2R-EVs significantly promoted the migration and invasion ability of CNE1 and CNE2 cells, respectively (Fig. 4 and Fig. 5), indicating that chemoradioresistant NPC cellderived EVs facilitated an aggressive phenotype of parental cells with different differentiation, and might involve in progress of NPC through regulating the invasion and metastasis of recipient cells.

\section{The chemoradiotherapy reshaped the IncRNAs profiling of EVs derived from the well and poorly differentiated NPC cells}

To further identify the DE IncRNAs regulated by chemoradiotherapy in EVs released by well and poor differentiated NPC cells, we performed the real-time Profiler PCR array. Between CNE1R-EVs vs CNE1-EVs and CNE2R-EVs vs CNER-EVs, there were 49 and 53 DE IncRNAs, respectively (Supplementary table S1 and S2). Overall, a total of 91 DE IncRNAs were affected by chemoradiotherapy. there were 15 upregulated DE IncRNAs found in the CNE2R-EVs, and 18 upregulated DE IncRNAs identified in the CNE1R-EVs, among which 4 upregulated DE IncRNAs were shared (Fig. 6A). Moreover, a total of 38 downregulated DE IncRNAs were identified in the CNE2R-EVs, and 31 downregulated DE IncRNAs were found in the CNE1R-EVs, among which 7 downregulated DE IncRNAs were shared (Fig. 6B). Hierarchical clustering was utilized to analyze the expressed pattern of the 11 shared IncRNAs (Fig. 6C). Chemoradioresistant NPC cells and parental cells had a high degree of heterogeneity. The similar spectral clustering was observed in NPC cells with or without receiving chemoradiotherapy.

\section{Discussion}

NPC is considered to be a distinct endemic disease in Southern China [1]. Chemoradiotherapy is a paramount approach as the main treatment strategy for NPC, but the effectiveness of chemoradiotherapy is limited for radiation resistance and drug resistance [23]. Chemotherapy can advance EV secretions in cancer cells, leading to the transfer of chemoresistance-related molecules to the adjacent cancer cells to induce susceptibility to chemotherapy $[24,25]$. To elucidate the functional effect of EVs released from chemoradioresistanct NPC cells, the chemoradioresistant CNE1R and CNE2R cells were established from their parental cell line. The chemoradioresistant NPC cell-derived EVs were internalized in parental cells, and promoted cell migration and invasion. Subsequently, to delineate the IncRNAs associated with chemoradioresistance, the IncRNA expression profile in EVs derived from chemoradioresistant NPC cells was revealed.

EVs may contribute to chemoradioresistance via transferring genetic information into sensitive cancer cells, and further affect the biological function (e.g. growth, migration, invasion and distant metastasis) of sensitive cancer cells $[26,27]$. For instance, recent literature elucidates that exosomal miR-20a-5p derived from radio-resistant NPC cells promoted the adjacent NPC cells radio-resistance through repression of Rab27B by targeting Rab27B 3'-UTR [28]. EBV infection-derived exosomes may activate the 
phosphoinositide 3-kinase/protein kinase B (PI3K/AKT) pathway to promote the stemness and chemoradioresistance of NPC through transferring LMP1 [29, 30]. NPC-exosomes modulates epithelial-tomesenchymal transition(EMT) of cells through delivering growth factor beta, resulting in resistance to chemotherapy [31, 32]. Here, chemoradioresistant NPC cells-derived EVs facilitated the migration and invasion ability of parental cells, suggesting these EVs promoted an aggressive phenotype of parental NPC cells, and might further affect chemoradioresistance and EMT of parental cells.

Several studies have shown that NPC-derived EVs carry various contents and play crucial roles in progression of NPC [33,34]. However, the effect of chemoradioresistant NPC-derived EVs on progression of NPC remains unknown via carrying IncRNAs. Linc-ROR facilitates NPC invasion and metastasis via enhancing EMT [35]. H19 has been verified to be involved in NPC metastasis [36]. Knockdown of XIST leads to a remarkable decrease of cell proliferation, a marked increase of radiosensitivity in NPC cells [37]. These findings suggest that chemoradioresistant NPC cells-derived EVs, containing active IncRNAs (i.e. MALAT1, FAM212B-AS1, LINC-PINT and H19), might exert functional role in NPC progression. This will be our future research direction.

\section{Conclusion}

In the present study, the chemoradioresistant NPC cells-derived EVs facilitated migration and invasion ability of cells with different differentiation. The 4 upregulated and 7 down-regulated IncRNAs were shared in EVs derived from well-differentiated and poor-differentiated NPC cells receiveing chemoradiotherapy. We propose this study provides the clue that secreted EVs of chemoradioresistant NPC cells may carry IncRNAs to affect aggressive phenotype of parental cells.

\section{Abbreviations}

Extracellular vesicles: EVs; nasopharyngeal carcinoma: NPC; long non-coding RNAs: IncRNA; Transmission Electron Microscopy:TEM; Nanoparticle Tracking Analysis: NTA; differentially expressed: $\mathrm{DE}$; plating efficiency: PE; Surviving fraction: SF.

\section{Declarations}

\section{Acknowledgements}

This project was supported by Yunnan provincial basic research program (2019FE001(-076)), Top Young Talents of the Ten Thousand Talents Program of Yunnan Province, Yunnan Health Science and Technology Project (2018NS0066), Scientific Research Project of Education Department of Yunnan Province (2018JS222).

\section{Competing interests}

The authors declare that they have no competing interests. 
Availability of data and material

The datasets used and/or analysed during the current study are available from the corresponding author on reasonable request.

\section{Code availability}

Not applicable.

\section{Authors' contributions}

Conceptualization and Funding acquisition, C.S. and Y.Y.; Data curation, W.X., R.S., C.L., and C.L.; Formal analysis, J.Q., Q.L., X.W., Q.W., G.X., and R.C.; Investigation, W.X., R.S., C.L., C.L., J.Q., Q.L., X.W., Q.W., G.X., and R.C.; Methodology, W.X., R.S., C.L., and C.L.; Writing - original draft, W.X., R.S., C.L., C.L., J.Q., Q.L., X.W., Q.W., G.X., and R.C.; Writing - review \& editing, W.X., R.S., C.L., and C.L.; All authors read and approved the final manuscript.

\section{Ethics approval}

Not applicable.

\section{Consent to participate}

All the authors listed have approved the manuscript.

\section{Consent for publication}

All the authors listed have approved the publication.

\section{References}

1. Lo KW, To KF, Huang DP. Focus on nasopharyngeal carcinoma. Cancer cell. 2004; 5: 423-8.

2. Tsang CM, Lui VWY, Bruce JP, Pugh TJ, Lo KW. Translational genomics of nasopharyngeal cancer. Semin Cancer Biol. 2020; 61:84-100.

3. Chen YP, Chan ATC, Le QT, Blanchard P, Sun Y, Ma J. Nasopharyngeal carcinoma. Lancet. 2019; 394: 64-80.

4. Razak AR, Siu LL, Liu FF, Ito E, O'Sullivan B, Chan K. Nasopharyngeal carcinoma: the next challenges. Eur J Cancer. 2010; 46: 1967-78.

5. Lo KW, Huang DP. Genetic epigenetic changes in nasopharyngeal carcinoma. Semin Cancer Biol. 2002; 12: 451-62.

6. Häusler M, Scheithauer S, Ritter K, Kleines M. Molecular diagnosis of Epstein-Barr virus. Expert Rev Mol Diagn. 2003; 3: 81-92. 
7. Zhao CX, Zhu W, Ba ZQ, et al. The regulatory network of nasopharyngeal carcinoma metastasis with a focus on EBV, IncRNAs and miRNAs. Am J Cancer Res. 2018; 8: 2185-209.

8. Kristensen CA, Kjaer-Kristoffersen F, Sapru W, Berthelsen AK, Loft A, Specht L. Nasopharyngeal carcinoma. Treatment planning with IMRT and 3D conformal radiotherapy. Acta oncologica. 2007; 46: 214-20.

9. Lee AW, Poon YF, Foo W, et al. Retrospective analysis of 5037 patients with nasopharyngeal carcinoma treated during 1976-1985: overall survival and patterns of failure. Int J Radiat Oncol Biol Phys. 1992; 23: 261-70.

10. Quinn JJ, Chang HY. Unique features of long non-coding RNA biogenesis and function. Nat Rev Genet. 2016; 17: 47-62.

11. Wu J, Hann SS. Functions and Roles of Long-Non-Coding RNAs in Human Nasopharyngeal Carcinoma. Cell Physiol Biochem. 2018; 45: 1191-204.

12. Li N, Yu J, Luo A, et al. LncRNA and mRNA signatures associated with neoadjuvant chemoradiotherapy downstaging effects in rectal cancer. J Cell Biochem. 2019; 120: 5207-217.

13. Xiong W, Jiang YX, Ai YQ, et al. Microarray Analysis of Long Non-coding RNA Expression Profile Associated with 5-Fluorouracil-Based Chemoradiation Resistance in Colorectal Cancer Cells. Asian Pac J Cancer Prev. 2015; 16: 3395-402.

14. Théry C. Exosomes: secreted vesicles and intercellular communications. F1000 Biol Rep. 2011; 3:15.

15. Shao H, Im H, Castro CM, Breakefield X, Weissleder R, Lee H. New Technologies for Analysis of Extracellular Vesicles. Chem Rev. 2018; 118: 1917-950.

16. Raposo G, Stoorvogel W. Extracellular vesicles: exosomes, microvesicles, and friends. J Cell Biol. 2013; 200: 373-83.

17. Tilghman RW, Hoover RL. The Src-cortactin pathway is required for clustering of E-selectin and ICAM1 in endothelial cells. FASEB J. 2002; 16: 1257-9.

18. Tilghman RW, Hoover RL. E-selectin and ICAM-1 are incorporated into detergent-insoluble membrane domains following clustering in endothelial cells. FEBS Lett. 2002; 525:83-7.

19. Lu J, Xu X, Liu X, et al. Predictive value of miR-9 as a potential biomarker for nasopharyngeal carcinoma metastasis. Br J Cancer. 2014; 110:392-8.

20. Lu J, Luo H, Liu X,et al. miR-9 targets CXCR4 and functions as a potential tumor suppressor in nasopharyngeal carcinoma. Carcinogenesis. 2014; 35: 554-63.

21. Cai L, Ye Y, Jiang Q, et al. Epstein-Barr virus-encoded microRNA BART1 induces tumour metastasis by regulating PTEN-dependent pathways in nasopharyngeal carcinoma. Nat Commun. 2015; 6: 7353.

22. Théry C, Witwer KW, Aikawa E, et al. Minimal information for studies of extracellular vesicles 2018 (MISEV2018): a position statement of the International Society for Extracellular Vesicles and update of the MISEV2014 guidelines. J Extracell Vesicles. 2018; 7: 1535750.

23. Chen C, Chen T, Huang C, Wang J, Fei Z. Experience of weekly cisplatin concurrent with intensitymodulated radiotherapy for locally advanced nasopharyngeal carcinoma patients with resistance to 
neoadjuvant chemotherapy. Medicine (Baltimore). 2017; 96: e8434.

24. Xiao X, Yu S, Li S, et al. Exosomes: decreased sensitivity of lung cancer A549 cells to cisplatin. PLoS One. 2014; 9: e89534.

25. Shedden K, Xie XT, Chandaroy P, Chang YT, Rosania GR. Expulsion of small molecules in vesicles shed by cancer cells: association with gene expression and chemosensitivity profiles. Cancer Res. 2003; 63: 4331-7.

26. Liu H, Wang J, Chen Y, et al. NPC-EXs Alleviate Endothelial Oxidative Stress and Dysfunction through the miR-210 Downstream Nox2 and VEGFR2 Pathways. Oxid Med Cell Longev. 2017; 2017: 9397631.

27. Shao Y, Shen Y, Chen T, Xu F, Chen X, Zheng S. The functions and clinical applications of tumorderived exosomes. Oncotarget. 2016; 7: 60736-51.

28. Huang D, Bian G, Pan Y, et al. MiR-20a-5p promotes radio-resistance by targeting Rab27B in nasopharyngeal cancer cells. Cancer Cell Int. 2017; 17: 32.

29. Yang CF, Yang GD, Huang TJ, et al. EB-virus latent membrane protein 1 potentiates the stemness of nasopharyngeal carcinoma via preferential activation of PI3K/AKT pathway by a positive feedback loop. Oncogene. 2016; 35: 3419-31.

30. Yang GD, Huang TJ, Peng LX, et al. Epstein-Barr Virus_Encoded LMP1 upregulates microRNA-21 to promote the resistance of nasopharyngeal carcinoma cells to cisplatin-induced Apoptosis by suppressing PDCD4 and Fas-L. PloS one. 2013; 8: e78355.

31. Acloque $H$, Thiery JP, Nieto MA. The physiology and pathology of the EMT. Meeting on the epithelialmesenchymal transition. EMBO Rep. 2008; 9: 322-6.

32. Andarawewa $\mathrm{KL}$, Erickson $\mathrm{AC}$, Chou WS, et al. lonizing radiation predisposes nonmalignant human mammary epithelial cells to undergo transforming growth factor beta induced epithelial to mesenchymal transition. Cancer Res. 2007; 67: 8662-70.

33. Becker A, Thakur BK, Weiss JM, Kim HS, Peinado H, Lyden D. Extracellular Vesicles in Cancer: Cell-toCell Mediators of Metastasis. Cancer cell. 2016; 30: 836-48.

34. Zhou Y, Xia L, Lin J, et al. Exosomes in Nasopharyngeal Carcinoma. J Cancer. 2018; 9: 767-77.

35. Li L, Gu M, You B, et al. Long non-coding RNA ROR promotes proliferation, migration and chemoresistance of nasopharyngeal carcinoma. Cancer Sci. 2016; 107: 1215-22

36. Li X, Lin Y, Yang X, Wu X, He X. Long noncoding RNA H19 regulates EZH2 expression by interacting with miR-630 and promotes cell invasion in nasopharyngeal carcinoma. Biochem Biophys Res Commun. 2016; 473: 913-9.

37. Han Q, Li L, Liang H, Li Y, Xie J, Wang Z. Downregulation of IncRNA X Inactive Specific Transcript (XIST) Suppresses Cell Proliferation and Enhances Radiosensitivity by Upregulating mir-29c in Nasopharyngeal Carcinoma Cells. Med Sci Monit. 2017; 23: 4798-807.

\section{Tables}

Page 12/16 
Table I. The radiobiological parameters of chemoradioresistant NPC cells (CNE1R and CNE2R) and parental cells.

\begin{tabular}{|llll|}
\hline Cells & Do(Gy) & Dq(Gy) & SF2 \\
CNE1 & 2.0333 & 1.2629 & 0.5810 \\
CNE1R & 2.3759 & 1.2926 & 0.6210 \\
CNE2 & 1.9539 & 0.4500 & 0.4290 \\
\hline CNE2R & 2.8114 & 1.3371 & 0.6620 \\
\hline
\end{tabular}

\section{Figures}

A

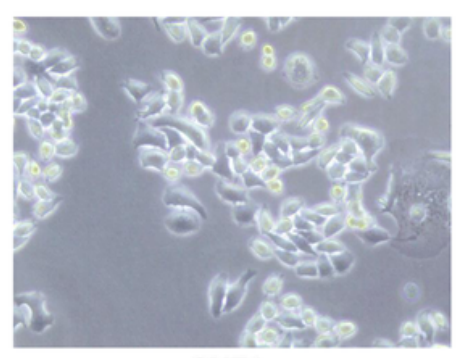

CNE1

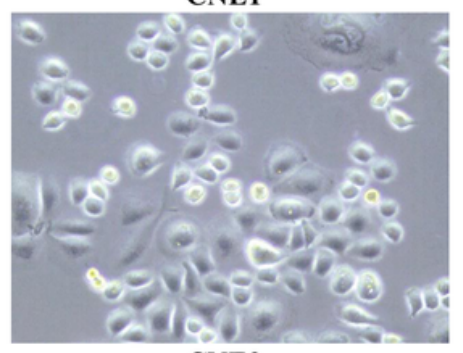

CNE2

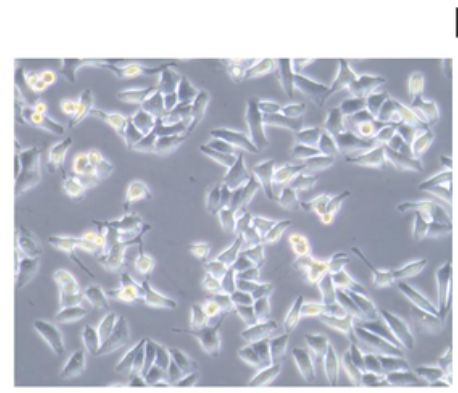

CNE1R

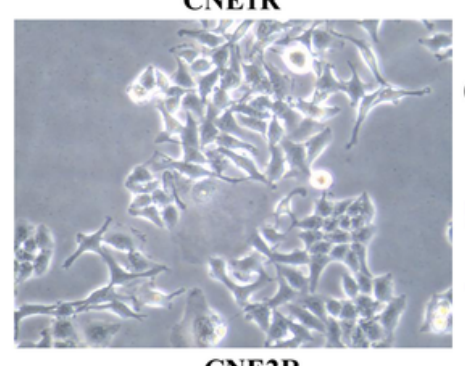

CNE2R
B

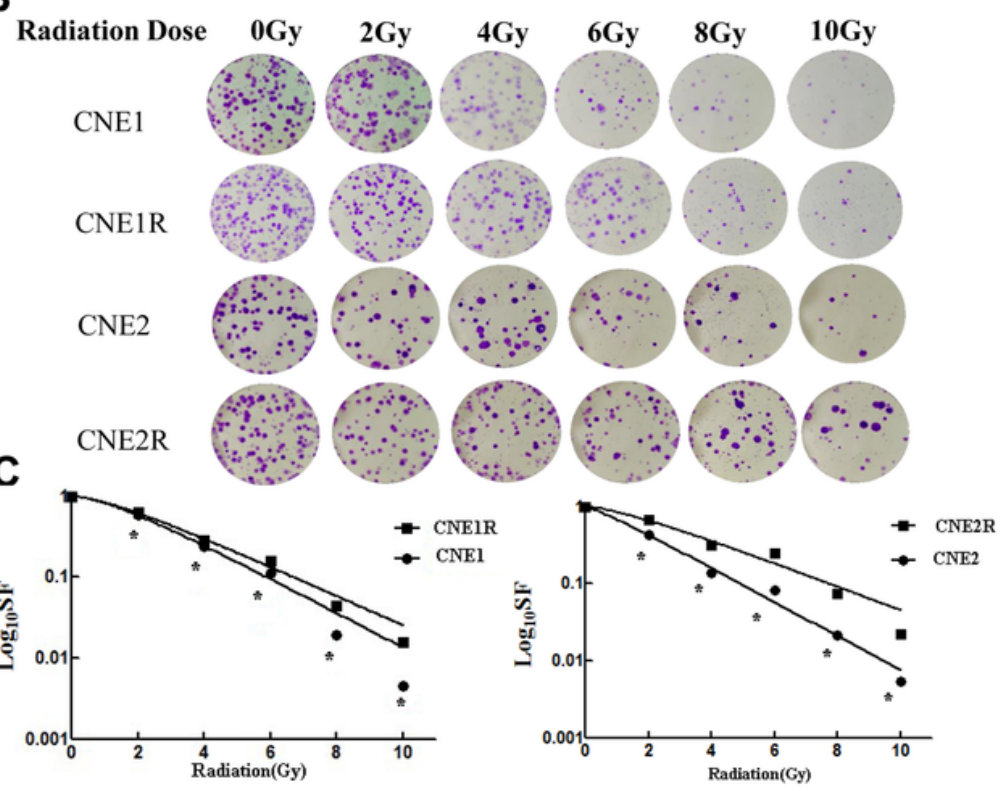

\section{Figure 1}

The chemoradioresistant NPC cells showed a higher concurrent chemoradioresistance compared to parental cells. (A) Morphological changes of parental cells (CNE1 and CNE2) and chemoradioresistant NPC cells (CNE1R and CNE2R) (Magnification, 10x). (B) The colony formation assay was used to determine the number of colonies of CNE1, CNE2, CNE1R and CNE2R. These cells were treated with $0.05 \mathrm{ug} / \mathrm{mL}$ cisplatin, and with gradient doses of irradiation (0 Gy, $2 \mathrm{~Gy}, 4 \mathrm{~Gy}, 6 \mathrm{~Gy}, 8 \mathrm{~Gy}$ and $10 \mathrm{~Gy}$ ) according to the gradient cell numbers $(300,400,800,1000,2000,4000$ cells/well). (C) Cell survival curve based on concurrent chemoradiotherapy. $S F$, surviving fraction. ${ }^{*} \mathrm{P}<0.05, \mathrm{n}=3$ in each group 
A
CNE1-EVs

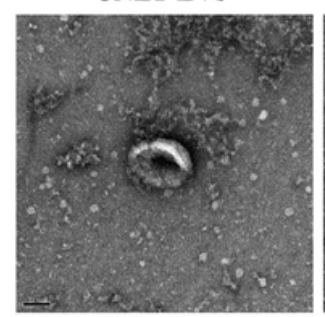

B

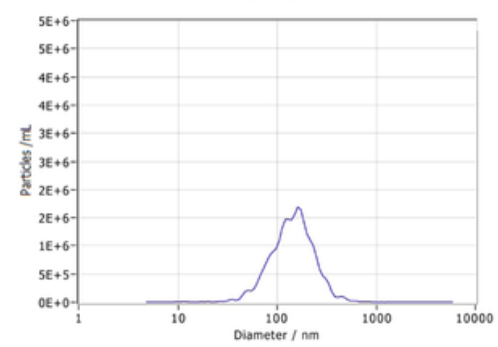

CNE1R-EVs

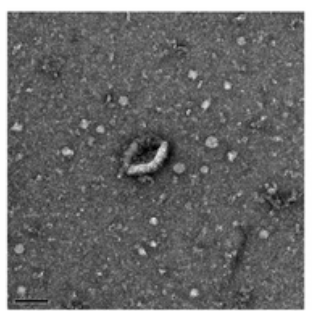

CNE2-EVs

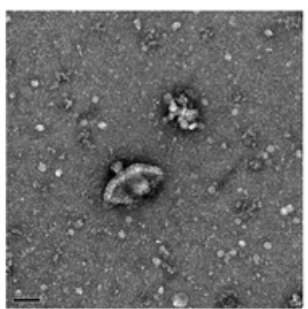

CNE2R-EVs

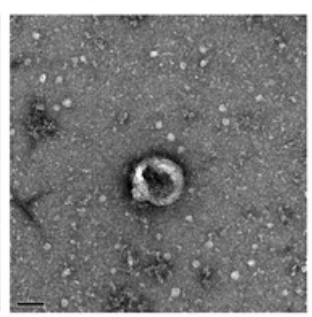

C

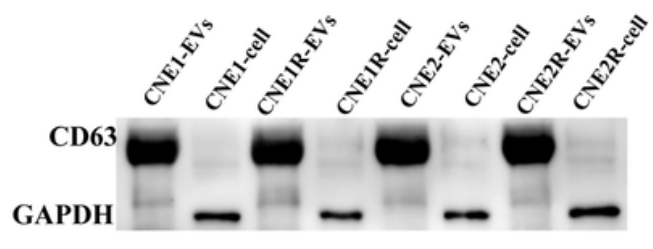

CNE2-EVs

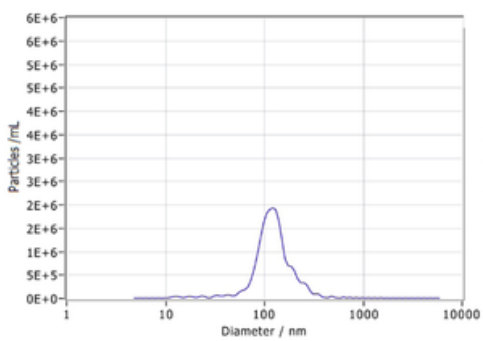

CNE2R-EVs

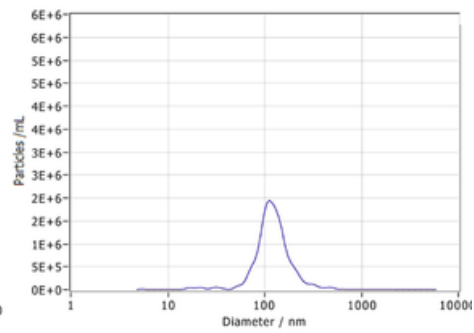

Figure 2

Characterization of extracellular vesicles (EVs) derived from well and poor- differentiated NPC cells with or without receiving chemoradiotherapy. (A) Transmission electron microscopy images of EVs derived from well-differentiated (CNE1 and CNE1R) and poor-differentiated NPC cells (CNE2 and CNE2R) with or without chemoradiotherapy, Scale bar, $100 \mathrm{~nm}$. (B) Secreted EVs of chemoradioresistant and parental NPC cells, including well differentiated and poorly differentiated cells, were analyzed for particle size by nanoparticle tracking analysis (NTA). (C) Western blot detected the expression of EV marker (CD63) in the secreted EVs of well-differentiated and poor-differentiated CNE1, CNE2, CNE1R and CNE2R

DiI

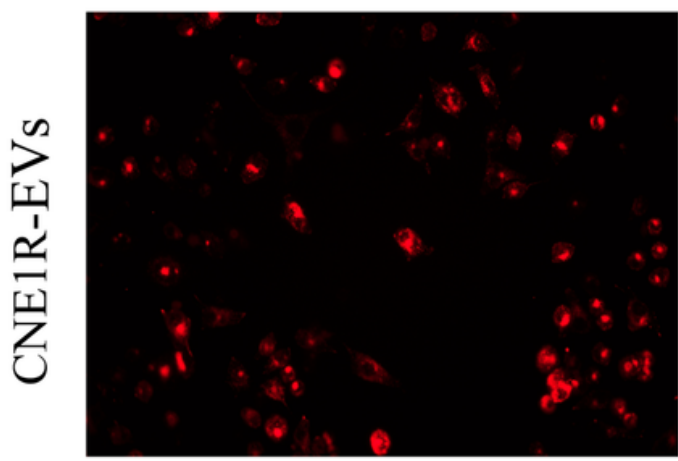

ש
DAPI
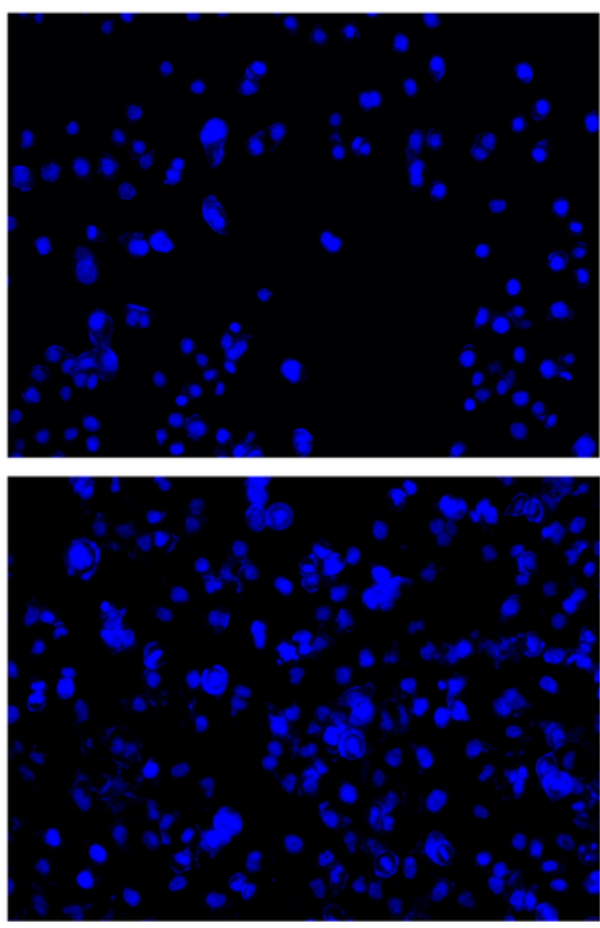

Merge
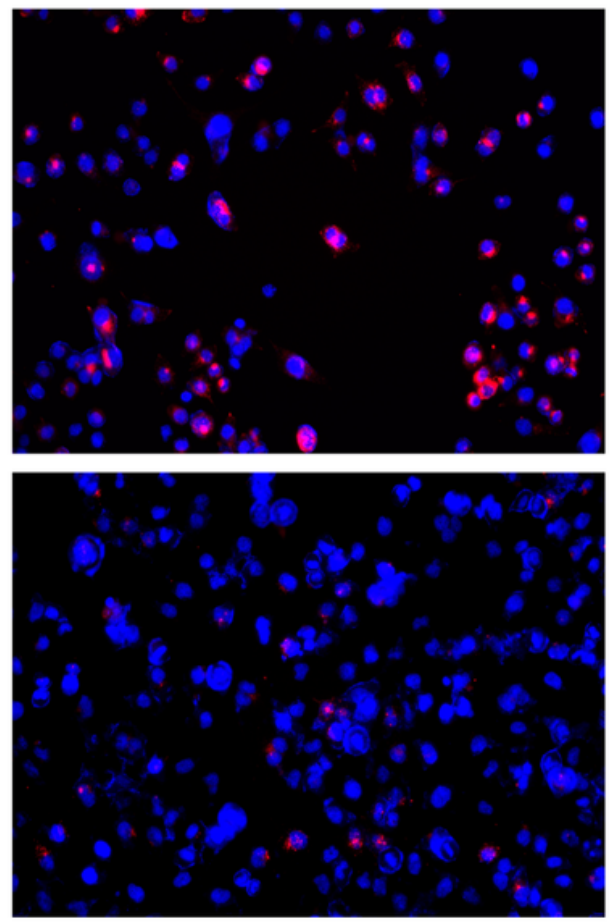


\section{Figure 3}

Internalization of EVs derived from chemoradioresistant NPC cells. Immunofluorescence images of Dillabelled EVs were taken up by CNE1 and CNE2 cells. The 100ul EVs derived from 5×106 CNE1R and CNE2R cells incubated the CNE1 and CNE2 cells for 24h, respectively. Magnification, $\times 40$

A

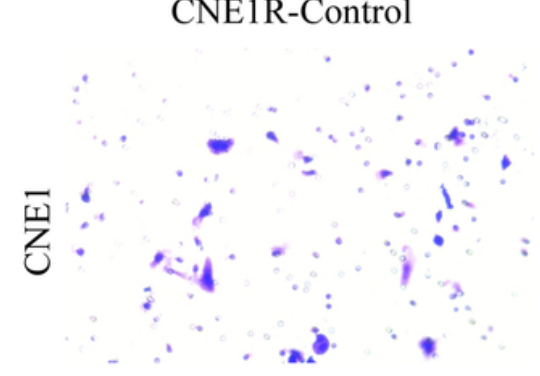

CNE2R-Control

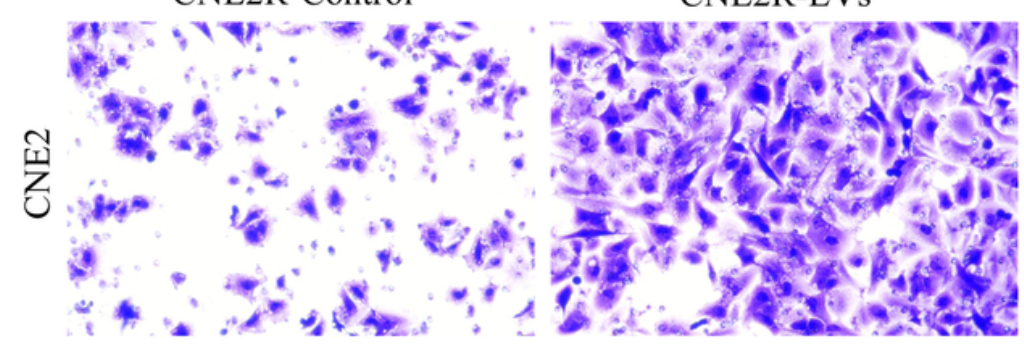

B

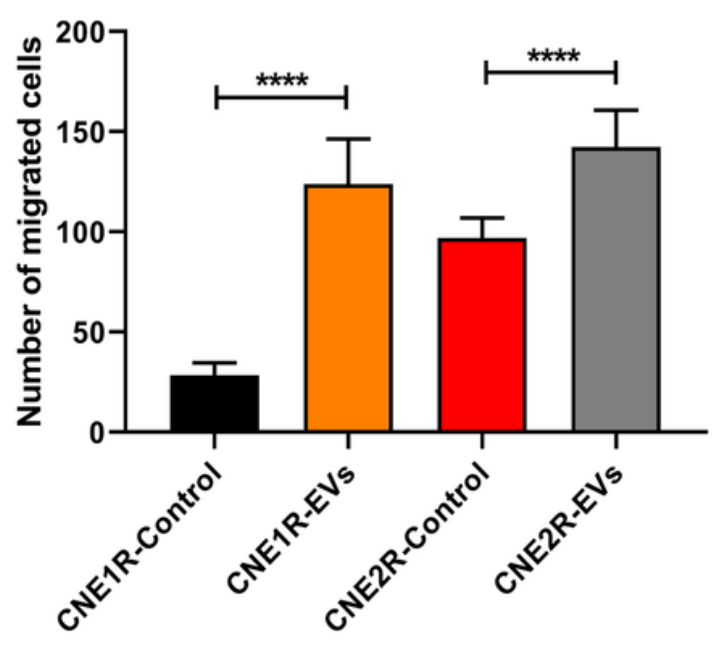

\section{Figure 4}

The chemoradioresistant NPC cells - derived EVs promoted migration ability of cells without receiving chemoradiotherapy. (A) Both in well-differentiated and poor-differentiated NPC cells, the chemoradioresistant NPC cells-derived EVs enhanced migration ability of cells without receiving chemoradiotherapy (B) A histogram showing the number of migrated cells The 100ul EVs derived from $5 \times 106$ CNE1R and CNE2R cells incubated the CNE1 and CNE2 cells for $24 \mathrm{~h}$, respectively. ${ }^{\star \star \star \star} P<0.0001$. $\mathrm{n}=3$ in each group

A
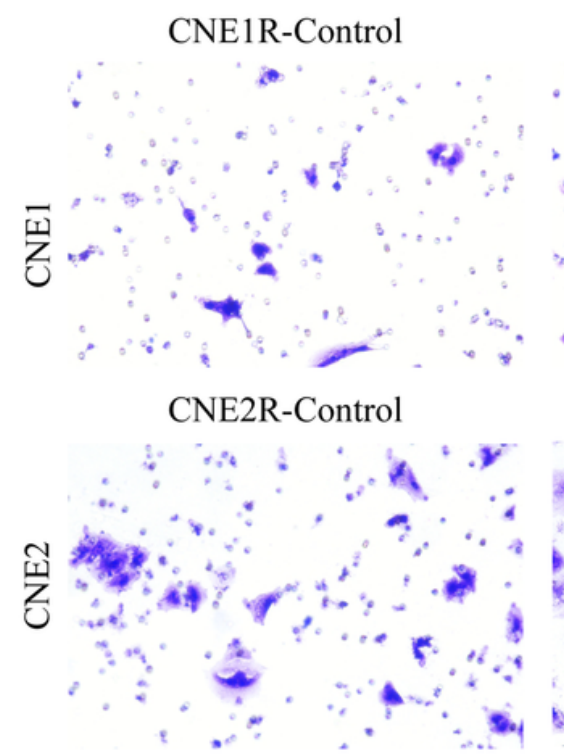

CNE1R-EVs

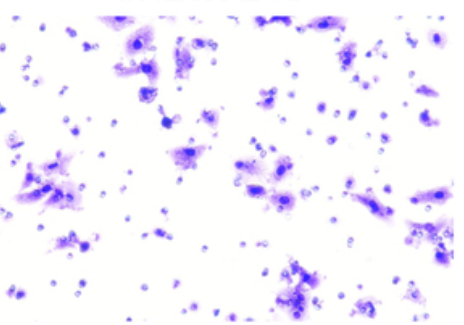

CNE2R-EVs

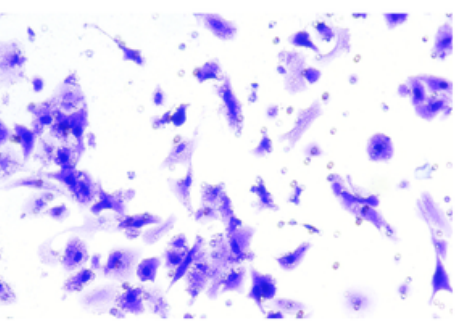

B

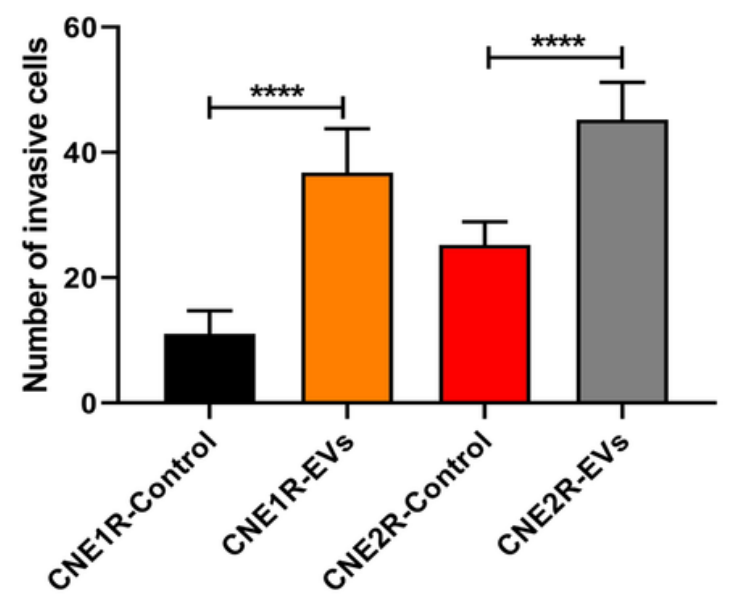


The secreted EVs of chemoradioresistant NPC cells facilitated invasive ability of parental cells. (A) Both in well-differentiated and poor-differentiated NPC cells, the secreted EVs of chemoradioresistant NPC cells promoted invasive ability of cells without receiving chemoradiotherapy. The 100ul EVs derived from $5 \times 106$ CNE1R and CNE2R cells incubated the CNE1 and CNE2 cells for 24h, respectively. (B) A histogram showing the number of invasive cells $* * * * P<0.0001$. $n=3$

A

CNE2R vs CNE2

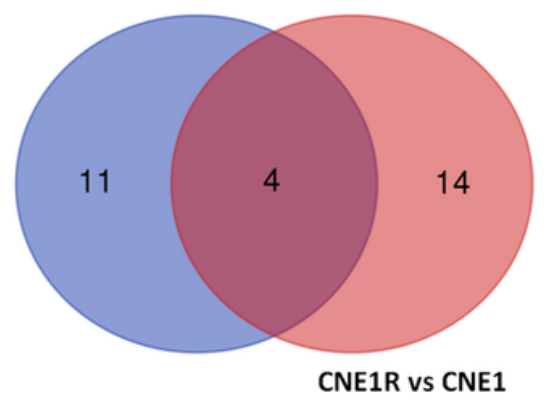

Up-regulated IncRNA

B

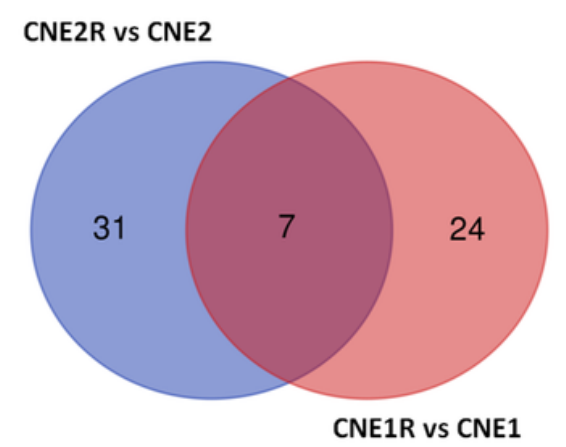

Down-regulated IncRNA
C

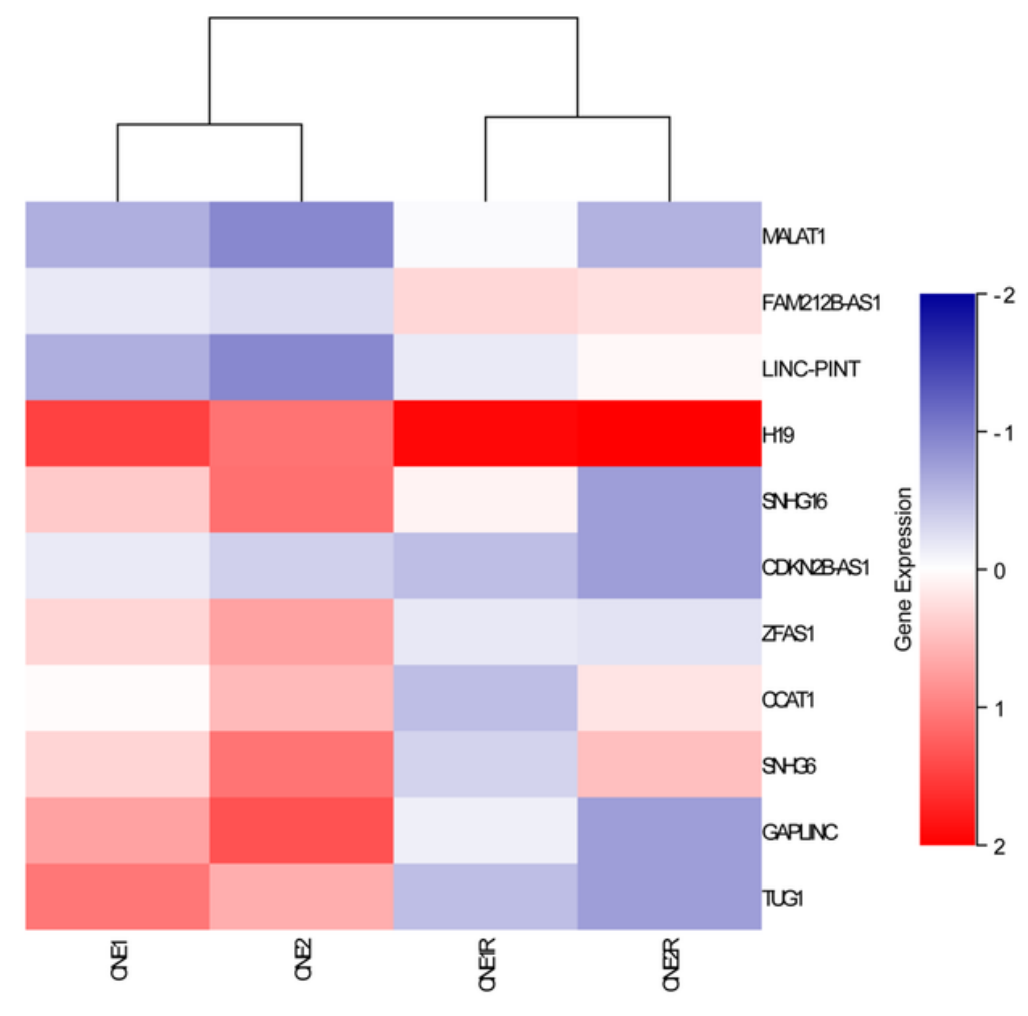

\section{Figure 6}

Differentially expressed (DE) long non-coding RNAs (IncRNAs) between EVs derived from parental and chemoradioresistant NPC cells. (A) Venn diagrams illustrating co-expressed up-regulated IncRNAs and co-expressed down-regulated IncRNAs (B) between CNE1 vs CNE2 and CNE1R vs CNE2R. (C) The heat map illustrating 11 DE IncRNAs in CNE1 and CNE2 cells, treated with or without chemoradiotherapy. The red indicates upregulation; the blue shows blue

\section{Supplementary Files}

This is a list of supplementary files associated with this preprint. Click to download.

- Tables1.xlsx

- Tables2.xlsx 